\title{
Mathematical Model and Stability Analysis of Inverter-Based Distributed Generator
}

\author{
Alireza Khadem Abbasi and Mohd Wazir Mustafa \\ Faculty of Electrical Engineering, Universiti Teknologi Malaysia, 81310 Johor Bahru, Johor, Malaysia \\ Correspondence should be addressed to Alireza Khadem Abbasi; alireza_khademabbasi2000@yahoo.com
}

Received 23 December 2012; Accepted 18 February 2013

Academic Editor: Vu Phat

Copyright (C) 2013 A. Khadem Abbasi and M. W. Mustafa. This is an open access article distributed under the Creative Commons Attribution License, which permits unrestricted use, distribution, and reproduction in any medium, provided the original work is properly cited.

\begin{abstract}
This paper presents a mathematical (small-signal) model of an electronically interfaced distributed generator (DG) by considering the effect of voltage and frequency variations of the prime source. Dynamic equations are found by linearization about an operating point. In this study, the dynamic of DC part of the interface is included in the model. The stability analysis shows with proper selection of system parameters; the system is stable during steady-state and dynamic situations, and oscillatory modes are well damped. The proposed model is useful to study stability analysis of a standalone DG or a Microgrid.
\end{abstract}

\section{Introduction}

Distributed generation (DG) systems have been expected to be an important electric power supply system for next generation. DGs are able to be installed near the loads, so they can increase the power quality and reliability of electricity delivered to sensitive loads. Some of the DG technologies require a power electronics interface in order to convert the energy into the grid compatible AC power. These interface devices make the sources more flexible in their operation and control compared to the conventional electrical machines. However, due to their negligible physical inertia, they also make the system potentially susceptible to oscillation resulting from network disturbances [1].

The coordinated operation and control of DGs together with loads and storage devices are central to the concept of microgrid [2]. The analysis of the dynamic stability of conventional power systems is well established, but for microgrid there is a need to investigate how circuit and control features give rise to particular oscillatory modes, and which of these have poor damping. Finding an exact mathematical model by considering DGs and their control is needed to investigate dynamic stability of the microgrid under transient events such as islanding from main grid and small-signal deviation like slow changing in load.

Reference [3] presented a small-signal model for inverter in stand-alone AC supply system by using only the droop controller's variables as state variables of the DG model. An averaged current source model has been suggested for the converter in [4]. In this investigation, the high frequency converter current dynamics have been neglected in order to focus exclusively on the dynamics and control of the islanded microgrid. Regardless of the type of the DG, an equivalent RLC circuit including output filter and transformers impedances has been modeled as a power circuit of DG in most of the literatures $[1,5-8]$. In these cases, output currents and voltages are considered as state variables. References $[1,6,7]$ by adding controller equations to the DG system made an accurate model for small signal stability analysis of a microgrid.

In most articles, DC part of the DG (DC filter's voltage and current) is not considered in dynamic studies, or it is assumed to be a constant value [6], so its effect is neglected in stability analysis. In the reference [8], the input DC voltage variation of the inverter has been represented as the external perturbation in the open-loop model of the DG, but it has been set as a constant during control loop design and frequency-domain analysis. The DC voltage has been related to the input and output equations of electronically interface of the DG by using switching function of rectifier and inverter in [5]. Moreover, two types of models have been presented in this paper for the prime source of the DG. While the proposed models have been used in steady-state and load flow 


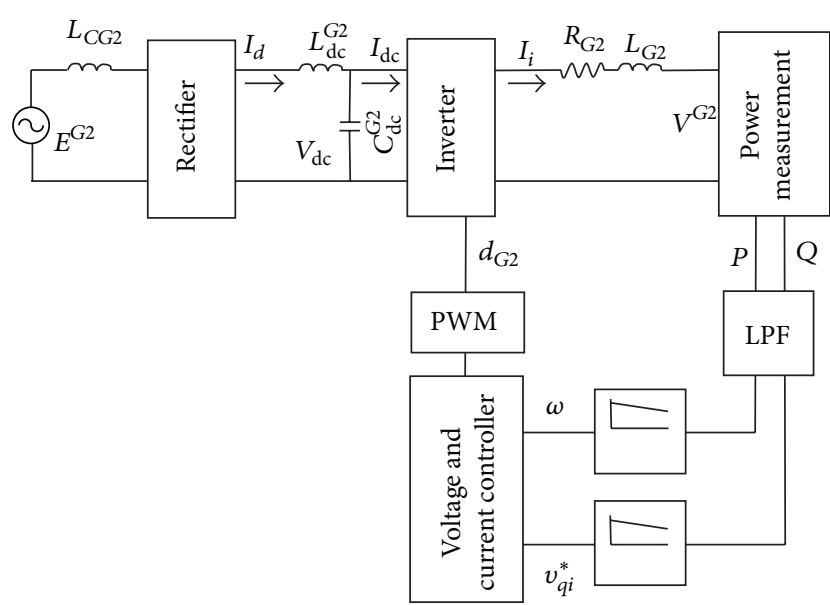

Figure 1: Single line equivalent of DG.

analysis, but their linear forms can be used also in dynamic studies.

The objective of this paper is to find a comprehensive dynamic model of DG, including the prime source, power electronically interfaces, output filter, and controller. The proposed model represents all components of the DG in a $d q 0$ reference frame, thus it ensures any application such as steady-state and dynamic analysis that meets requirements and constraints of both AC and DC parts of the system.

The dynamic stability of the DG is investigated by the small signal and step response analysis. In this paper, the DG is connected only to the static load, but it can be extended into the network or typical microgrid for more applications.

\section{System Studied}

2.1. Power Circuit. A simplified single-line diagram of the studied DG is illustrated in Figure 1 . DG is included of a three-phase source, a three-phase rectifier, an inverter, and a three-phase static load on the output bus. The DG source can be representatives of wind turbine or microturbine generator for medium and low voltage levels, respectively. Regardless of the power measurement and control loops, the system shown in Figure 1 can be studied as a power circuit of DG in microgrid stability analysis.

2.2. Control System. As shown in Figure 1, the control of DG included power, voltage, and current controllers. The power control has been achieved by applying droop control for both active and reactive powers. The droop control for an electronically interfaced DG has the role of the governor for a synchronously generator. Where, by an increase in load, the reference frequency is decreased. In the similar way, the reactive power is controlled by a droop characteristic in the voltage magnitude.

In this study, the sin-triangle modulation strategy is used to prepare applied voltage in the converter. The next parts of the control system are the outer voltage and inner current control loops, which are designed to reject high-frequency disturbances and provide sufficient damping for the output filter by using the proportional integral (PI) compensators.

\section{System Equations}

3.1. Power Circuit. The dynamic model of AC side of DG in three-phase $a b c$ frame is obtained from

$$
v_{i_{a b c}}=R_{G 2} i_{i_{a b c}}^{G 2}+L_{G 2} \rho i_{i_{a b c}}^{G 2}+v_{a b c}^{G 2},
$$

where $i_{i_{a b c}}^{G 2}, v_{a b c}^{G 2}$, and $v_{i_{a b c}}$ are vectors of the instantaneous amounts of the output currents, bus voltages, and the converter output voltages, respectively. Then (1) is transformed to the $d-q$ reference frame of DG that rotates at frequency $\omega_{G 2}$ related to the angular velocity of the voltage space vector of the bus. The transferred equations are

$$
E_{1}^{G 2} \rho i_{q d_{i}}^{G 2}=A_{1}^{G 2} i_{q d_{i}}^{G 2}+v_{q d_{i}}^{G 2}-v_{q d}^{G 2},
$$

where $\rho$ is the operator $d / d t$.

3.2. DC Interface Equations. The average voltage and current of capacitor and inductance of dc filter will change with time during transient. For the rectifier under operation without phase delay and with commutating inductance, we have [9]

$$
\bar{V}_{d}^{G 2}=\frac{3 \sqrt{3}}{\pi} E_{q}^{G 2}-\frac{3}{\pi} \frac{X_{L_{C G 2}}}{\omega_{b}} \omega_{E} I_{d}^{G 2}-\frac{2}{\omega_{b}} X_{L_{c G 2}} \rho I_{d}^{G 2} .
$$

We also have

$$
X_{L_{d c G 2}} \rho I_{d}^{G 2}=\bar{V}_{d}^{G 2}-V_{d c}^{G 2}
$$

From (3) and (4),

$$
\begin{aligned}
V_{d c}^{G 2}= & \frac{3 \sqrt{3}}{\pi} E_{q}^{G 2}-\frac{3}{\pi} \frac{X_{L_{c G 2}}}{\omega_{b}} \omega_{E} I_{d}^{G 2} \\
& -\frac{1}{\omega_{b}}\left(X_{L_{d c G 2}}+2 X_{L_{c G 2}}\right) \rho I_{d}^{G 2} .
\end{aligned}
$$

Equation (5) expresses the relationship between dynamic parts of the dc filter and DG source voltage. The $\omega_{E}$ is the frequency of the source, and $E_{q}^{G 2}$ is the network side voltage's $q$ component. The voltage is wrote in the form of

$$
\rho V_{d c}^{G 2}=\omega_{b} X_{c_{d c G 2}}\left(I_{d}^{G 2}-I_{d c}^{G 2}\right)
$$

Apart from the harmonics in the voltage waveform and considering sin-triangle modulation for inverter, voltages become

$$
\begin{gathered}
v_{q_{i}}^{G 2}=\frac{d_{G 2}}{2} V_{d c}^{G 2}, \\
v_{d_{i}}^{G 2}=0,
\end{gathered}
$$

where $d_{G 2}$ is duty cycle.

We will see that the $q$ component of converter voltage is controlled by controller and $v_{d i}^{G 2}$ is considered to be zero. 


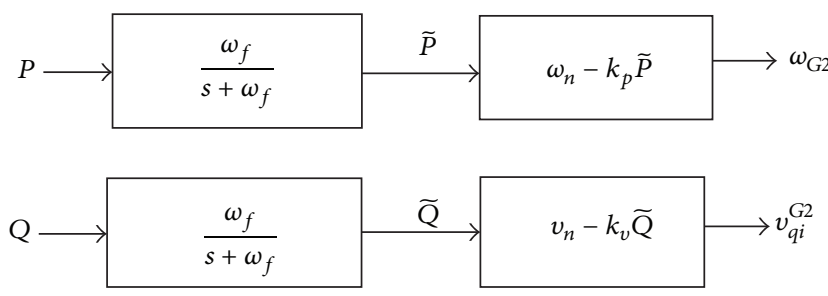

FIGURE 2: Inverter power controller.

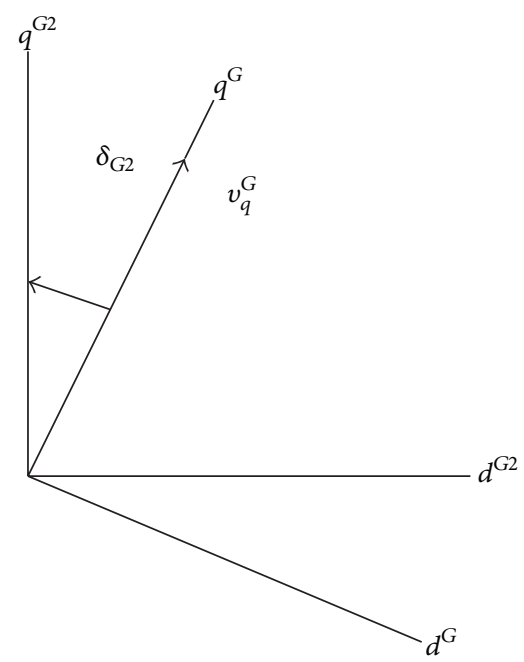

FIGURE 3: Common reference frame.

The instantaneous power balance between two sides of the inverter is established by

$$
V_{d c}^{G 2} I_{d c}^{G 2}=\frac{3}{2} v_{q i}^{G 2} i_{q i}^{G 2} .
$$

According to (7), we have

$$
I_{d c}^{G 2}=\frac{3 d_{G 2}}{4} i_{q i}^{G 2} .
$$

Rewriting (6) with regard to (9),

$$
\rho V_{d c}^{G 2}=\omega_{b} X_{C_{d c G 2}}\left(I_{d}^{G 2}-\frac{3 d_{G 2}}{4} i_{q i}^{G 2}\right) .
$$

The dynamic equations of DC part are represented by (5) and (10).

3.3. Control System Equations. Figure 2 shows inverter power controller, where $P$ and $Q$ are measured values of inverter active and reactive output powers. Moreover, $\widetilde{P}$ and $\widetilde{Q}$ are filtered values of power after passing through the low-pass filter. As shown in Figure 3, the inverter output voltage $v_{i}^{\mathrm{G} 2}$ and inverter output frequency $\omega_{\mathrm{G} 2}$ are adjusted by the droop controller characteristics described by (11) and (12), respectively, as follows:

$$
\begin{aligned}
& \omega_{G 2}=\omega_{n}-k_{p} \widetilde{P}, \\
& v_{q i}^{G 2 *}=v_{n}-k_{v} \widetilde{Q} .
\end{aligned}
$$

Here, $\omega_{n}$ and $v_{n}$ represent the nominal frequency and $q$-axis output voltage set points, respectively. $v_{q i}^{\mathrm{G} 2 *}$ is the duty cycle $\left(d_{G 2}\right)$.

The power measurement outputs are given by (13) and (14), where $\omega_{f}$ is the cutoff frequency of the low-pass filter and $s$ is the Laplace operator

$$
\begin{aligned}
& \widetilde{P}=\frac{\omega_{f}}{s+\omega_{f}} P, \\
& \widetilde{Q}=\frac{\omega_{f}}{s+\omega_{f}} Q .
\end{aligned}
$$

3.4. Common Reference Frame Theory. All equations of DG have been written in its reference frame. In power system analysis, it is necessary to relate all components' reference frames to a common frame. This common reference frame would be represented as constant voltages in the synchronous reference frame. In microgrid studies, DG with the biggest nominal power is considered as a common reference frame.

Figure 3 shows the common reference frame of a microgrid by $q$ and $d$ axis rotating at the synchronous angular velocity of $\omega_{e}$. The $q$ axis is in orientation of the voltage vector of the reference DG, $v_{q}^{G}$. By assuming $v_{q}^{\mathrm{G} 2}$ on the $q$ axis of the DG2 reference frame, $q^{G 2}, \delta_{G 2}$ is the angle between the DG2 reference frame and common reference frame. In order to transfer variables in the DG2 reference frame to common reference frame, the transformation matrix is defined as

$$
\begin{gathered}
f^{G}=T_{G 2} f^{G 2}, \quad f^{G 2}=T_{G 2}^{-1} f^{G}, \\
T_{G 2}=\left[\begin{array}{cc}
\cos \delta_{G 2}^{0} & \sin \delta_{G 2}^{0} \\
-\sin \delta_{G 2}^{0} & \cos \delta_{G 2}^{0}
\end{array}\right], \\
T_{G 2}^{-1}=\left[\begin{array}{cc}
\cos \delta_{G 2}^{0} & -\sin \delta_{G 2}^{0} \\
\sin \delta_{G 2}^{0} & \cos \delta_{G 2}^{0}
\end{array}\right] .
\end{gathered}
$$

To connect an inverter to the whole system the output variables that have the connection with the network need to be converted to the common reference frame. In this case the output variables are the output currents $i_{q d i}^{G 2}$ and the network side voltages $v_{q d}^{G 2}$. Because the dynamic of one DG is studied in this paper, the $\delta_{G 2}$ is zero, and transformation matrix is the unit.

3.5. Small-Signal Model. By liberalization of nonlinear differential equations around the operating steady-state point, the small-signal model of the system has been created [9].

For inverter equations, first output variables must be transferred to the common reference frame and then be linearized. By using (15) inverter output currents and voltages in the common reference frame can be achieved as

$$
\begin{aligned}
& i_{q d i}^{G 2}=T_{G 2}^{-1} i_{q d i}^{G}, \\
& v_{q d}^{G 2}=T_{G 2}^{-1} v_{q d}^{G} .
\end{aligned}
$$


Then with linearization technique, we have

$$
\begin{aligned}
& \Delta i_{q d i}^{G 2}=T_{G 2}^{-1} \Delta i_{q d i}^{G}+i_{\delta_{G 2}}^{0} \Delta \delta_{G 2}, \\
& \Delta v_{q d}^{G 2}=T_{G 2}^{-1} \Delta v_{q d}^{G}+v_{\delta_{G 2}}^{0} \Delta \delta_{G 2},
\end{aligned}
$$

where

$$
\begin{aligned}
& i_{\delta_{G 2}^{0}}^{0}=\left[\begin{array}{ll}
-i_{d i 0}^{G 2} & i_{q i 0}^{G 2}
\end{array}\right]^{T}, \\
& v_{\delta_{G 2}}^{0}=\left[\begin{array}{ll}
-v_{d 0}^{G 2} & v_{q 0}^{G 2}
\end{array}\right]^{T} .
\end{aligned}
$$

The subscript 0 denotes steady-state quantities. The $\Delta \delta_{G 2}$ could be rewritten as a function of output voltages in common reference frame as follows

$$
\delta_{G 2}=\tan ^{-1}\left(\frac{v_{d}^{G}}{v_{q}^{G}}\right) .
$$

With linearization of (21),

$$
\Delta \delta_{G 2}=m_{q_{\delta G 2}} \Delta v_{q}^{G}+m_{d_{\delta G 2}} \Delta v_{d}^{G},
$$

where

$$
\begin{aligned}
& m_{q_{\delta G 2}}=-\frac{v_{d 0}^{G}}{v_{q 0}^{G}+v_{d 0}^{G}}, \\
& m_{d_{\delta G 2}}=\frac{v_{q 0}^{G}}{v_{q 0}^{G}+v_{d 0}^{G}} .
\end{aligned}
$$

For more information, see $[3,7]$. Furthermore, the linear equation of the inverter output voltage could be written as

$$
\Delta v_{q_{i}}^{G 2}=\frac{d_{G 2_{0}}}{2} \Delta V_{d c}^{G 2}+\frac{V_{d c 0}^{G 2}}{2} \Delta d_{G 2} .
$$

By replacing (7) and (18) into the inverter equations and linearization with considering (19), (22), and (24) the linear equations of the DG have been arranged in the basic style of the linear differential equations as

$$
\begin{gathered}
\rho \Delta X^{\mathrm{DG}}=A^{\mathrm{DG}} \Delta X^{\mathrm{DG}}+B^{\mathrm{DG}} \Delta U^{\mathrm{DG}}, \\
\Delta X^{\mathrm{DG}}=\left[\begin{array}{ll}
\Delta X^{G 2} & \Delta X_{C}^{G 2}
\end{array}\right], \\
\Delta U^{\mathrm{DG}}=\left[\begin{array}{ll}
\Delta U^{G 2} & \Delta U_{C}^{G 2}
\end{array}\right],
\end{gathered}
$$

where $\Delta U^{\mathrm{DG}}$ and $\Delta X^{\mathrm{DG}}$ are control and state variables respectively. The state matrix of the system is expressed by $A^{\mathrm{DG}}$ that is used in dynamic analysis of the DG.

The output vector of the system is defined as a linear combination of control and state variables in the form of

$$
\Delta Y^{\mathrm{DG}}=C \Delta X^{\mathrm{DG}}+D \Delta U^{\mathrm{DG}} .
$$

In the power system small signal stability analysis, the current injection into the network from the device is considered as the output signal [10]. For the DG, the output current is one of the state variables, so we have

$$
\Delta i_{q d i}^{G}=C^{\mathrm{DG}} \Delta X^{\mathrm{DG}} .
$$

All matrices are available in Appendix A in detail.

Finally, the transfer function is defined by

$$
\frac{\Delta Y^{\mathrm{DG}}}{\Delta U^{\mathrm{DG}}}=C^{\mathrm{DG}}\left(S I-A^{\mathrm{DG}}\right)^{-1} B^{\mathrm{DG}}+D^{\mathrm{DG}} .
$$

3.6. Eigenvalue Analysis. In power systems small-signal stability analysis is aimed to determine the properties of operation parameter variations that are independent from disturbance intensity. Eigenvalue analysis is used to show the information of different stability modes for power system small-signal stability problems. A system is stable when all of its modes are stable. Furthermore, it is required that all oscillations are well and quickly damped. This technique is declared in [11] in details.

In this paper, the participation factors and eigenvalues of the state matrix are computed and analyzed. Eigenvalues with negative real parts show that the system is stable. the state variable with the highest normalized participation factor is the best choice for feedback signal if it may be a measurable physical variable.

\section{Case Study and Results}

To study small-signal stability of the model, a $10 \mathrm{kVA}$ DG connected to a $9 \mathrm{~kW}$ static load is considered as the case study. All DG information is taken from [1]. The output impedance of DG is $0.1+j 0.4239 \Omega$. The cutoff frequency $\omega_{f}$ is considered $31.41 \mathrm{Rad} / \mathrm{s}$.

The rectifier's input inductance $X_{L_{c}}$ is used to amend the input current. The inductor with low value may not restrict the peak current, and its high amount may reduce the input voltage [10]. Hence, a $1.06(\mathrm{mH})$ inductor is chosen as an optimized value.

The DC filter is involved in a small series inductance, $X_{L_{d c}}$, (here $0.03 \mathrm{mH}$ ) and a shunt capacitance, $X_{c_{d c}}$, to smooth the diode rectifier output. With regard to [10], a $165 \mu \mathrm{F} / \mathrm{kW}$ capacitance is selected in this study. For DG, we have

$$
\text { Power }=10 \mathrm{~kW} \text {. }
$$

Hence

$$
C=165 * 100=1650 \mu \mathrm{F} \text {. }
$$

The steady-state values of the system are calculated by load flow program.

In order to realize the sensitivity and dynamic behaviour, eigenvalues trajectory plots are drawn as a function of some system parameters. Figure 4 shows the locations of eigenvalues as a function of variation of $k_{p}$ from 0.00000004 to 0.000094 with the rate of 0.000001 . In this case, $k_{v}$ is equal to 0.0013 .

Figure 5 shows the loci of eigenvalues corresponding to variation of $k_{v}$ from 0.00003 to 0.1 with the rate of $0.001 . k_{p}$ is considered to be 0.000015 . 


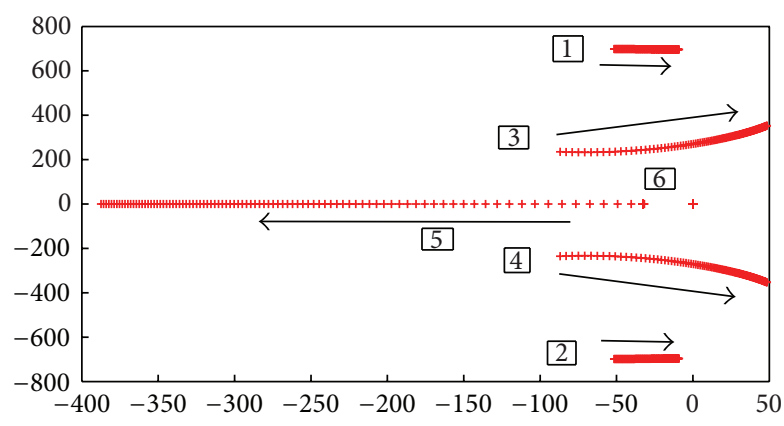

FIGURE 4: Loci of eigenvalues according to kp changing.

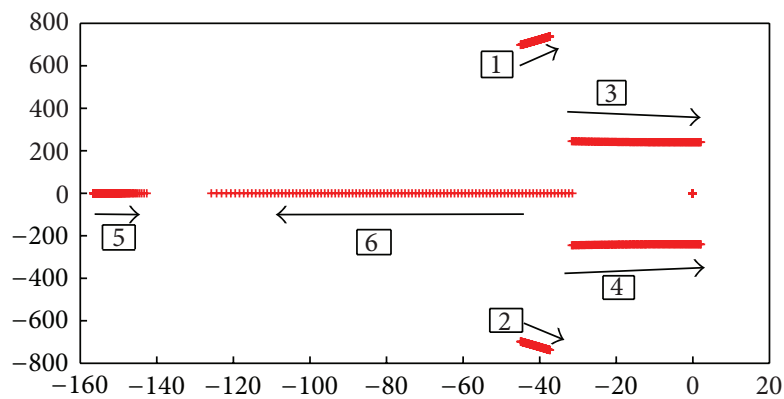

FIGURE 5: Loci of eigenvalues according to kv changing.

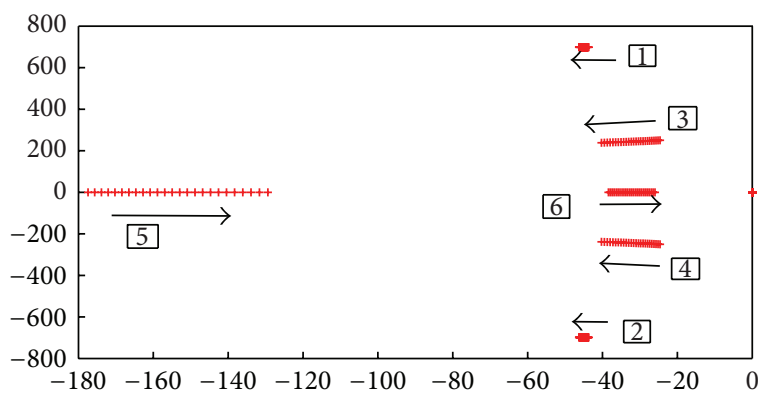

FIGURE 6: Loci of eigenvalues according to wf changing.

Trajectory plots show instability in modes of $1,2,3$, and 4 if the $k_{p}$ and $k_{v}$ increase to big values.

With regard to $k_{p}$ and $k_{v}$ as constant values of 0.000015 and 0.0013 , Figure 6 shows the root locus plot for the model as a function of the filter cutoff frequency $\omega_{f}$ from 37 to 25 with the rate of -0.5 . It can be seen that eigenvalues 5 and 6 move to right side of the plot.

Results are used to adjust the gains of the power controller. With optimal values of controller gains and appropriate selection of the DG parameters, all oscillatory modes are well damped, and the proposed model is stable.

By using QR-decomposition method in the MATLAB software environment, the eigenvectors and eigenvalues of the state matrix have been computed in operating point. The DG system along with its controller is described by six eigenvalues: $\lambda_{1,2}=-44.90 \pm 698.37 i, \lambda_{3,4}=-31.14 \pm$ 244.66i, $\lambda_{5}=-156.58$, and $\lambda_{6}=-32.62$.
TABLE 1: Participation factors.

\begin{tabular}{ccccccc}
\hline & $\lambda_{1}$ & $\lambda_{2}$ & $\lambda_{3}$ & $\lambda_{4}$ & $\lambda_{5}$ & $\lambda_{6}$ \\
\hline$\Delta i_{q i}^{G 2}$ & 0.2428 & 0.2428 & 0.2001 & 0.2001 & 0.0781 & 0.0 \\
$\Delta i_{d i}^{G 2}$ & 0.0497 & 0.0497 & 0.3646 & 0.3646 & 0.0829 & 0.0 \\
$\Delta v_{d c}^{G 2}$ & 0.4458 & 0.4458 & 0.0528 & 0.0528 & 0.0 & 0.0 \\
$\Delta I_{d}^{G 2}$ & 0.2522 & 0.2522 & 0.2025 & 0.2025 & 0.0463 & 0.0 \\
$\Delta \omega_{G 2}$ & 0.0094 & 0.0094 & 0.1782 & 0.1782 & 0.7923 & 0.0005 \\
$\Delta d_{G 2}$ & 0.0001 & 0.0001 & 0.0019 & 0.0019 & 0.0004 & 0.9995 \\
\hline
\end{tabular}

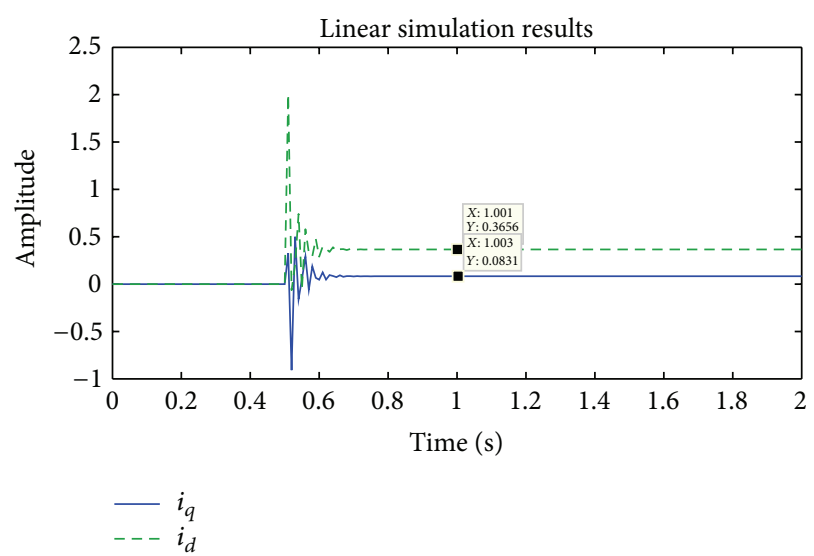

FIGURE 7: Step response of current to the source voltage.

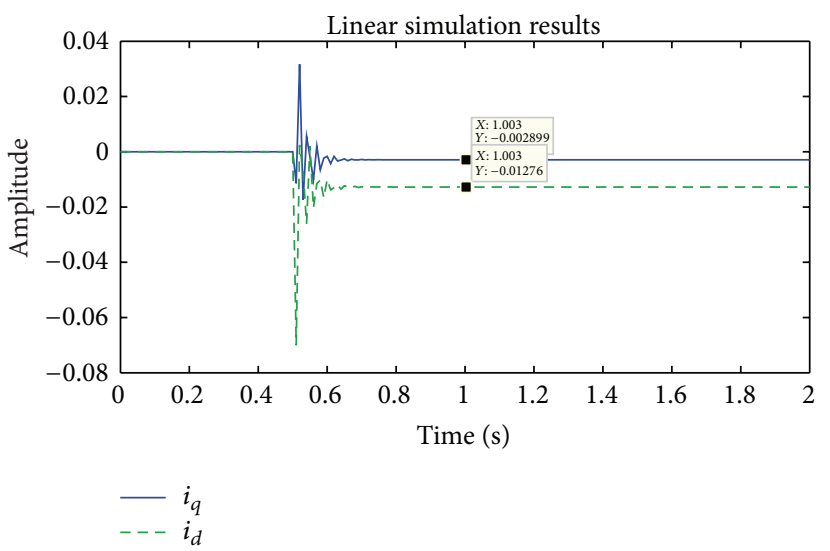

FIGURE 8: Step response of current to the source angular velocity.

The normalized participation factors of the dynamic model are represented in Table 1. It is a useful indication of the role of a state on the mode. As seen in oscillatory modes of $1,2,3$, and 4 , the state variables of DC part have the main role.

Figures 7 and 8 show step responses of the output current according to the source voltage and frequency. The small steady-state error in $i_{q_{i}}$ is caused by the effects of them on DC voltage and DC current. The variation in $i_{d_{i}}$ is due to coupling between $q$ and $d$ components of current. By control of rectifier and making a constant DC voltage this effects will be removed. Error specifications also could be adjusted by adding current and voltage control loops. 


\section{Conclusion}

The mathematical model of the inverter-based DG has been proposed in this paper. The proposed model has included power and control circuits of the DG. The dynamics of the DC filter and the effects of the prime source have been also considered in the DG model. By using eigenvalue analysis, the stability of proposed model has been studied in detail. This study presented that all oscillatory modes are well damped with suitable selection of system parameters. Since the proposed model is stable, it can be used in the dynamic and steady-state study of a microgrid.

\section{Appendices}

Linear equations of the DG.

\section{A. Power Circuit}

A.1. AC Part. By substituting (18) into the (2) and linearization

$$
E_{1}^{G 2} \Delta \dot{i}_{q d_{i}}^{G}=A_{1}^{G 2} \Delta i_{q d_{i}}^{G 2}+B_{1}^{G 2} \Delta v_{q d_{i}}^{G 2}+B_{2}^{G 2} \Delta v_{q d}^{G}+B_{3}^{G 2} \Delta \omega_{G 2}
$$

where

$$
\begin{gathered}
E_{1}^{G 2}=\left[\begin{array}{cc}
\frac{X_{l_{G 2}}}{\omega_{b}} & 0 \\
0 & \frac{X_{l_{G 2}}}{\omega_{b}}
\end{array}\right], \quad A_{1}^{G 2}=\left[\begin{array}{cc}
-R_{G 2} & -\frac{\omega_{G 2} X_{l_{G 2}}}{\omega_{b}} \\
\frac{\omega_{G 2_{0}} X_{l_{G 2}}}{\omega_{b}} & -R_{G 2}
\end{array}\right], \\
B_{1}^{G 2}=T_{G 2}\left[\begin{array}{l}
1 \\
0
\end{array}\right], \\
B_{2}^{G 2}=\left[\left(T_{G 2} A_{1}^{G 2} i_{\delta_{G 2}}^{0}-T_{G 2} v_{\delta_{G 2}}^{0}\right) M_{q d}^{G 2}-I\right] \\
B_{3}^{G 2}=-T_{G 2} E_{1}^{G 2} i_{\delta_{G 2}^{0}}^{0}, \quad M_{q d}^{G 2}=\left[m_{q_{\delta G 2}} m_{d_{\delta G 2}}\right] \\
\Delta \omega_{G 2}=\Delta \dot{\delta}_{G 2} .
\end{gathered}
$$

By (23) and $\Delta v_{d_{i}}^{G 2}=0$

$$
\begin{aligned}
E_{1}^{G 2} \Delta \dot{i}_{q d_{i}}^{G}= & A_{1}^{G 2} \Delta i_{q d_{i}}^{G}+B_{1}^{G 2} \frac{d_{G 2_{0}}}{2} \Delta V_{d c}^{G 2}+B_{1}^{G 2} \frac{V_{d c 0}^{G 2}}{2} \Delta d_{G 2} \\
& +B_{2}^{G 2} \Delta v_{q d}^{G}+B_{3}^{G 2} \Delta \omega_{G 2}, \\
E_{1}^{G 2} \Delta \dot{i}_{q d_{i}}^{G}= & A_{1}^{G 2} \Delta i_{q d_{i}}^{G}+F_{1}^{G 2}\left[\begin{array}{c}
\Delta V_{d c}^{G 2} \\
\Delta I_{d}^{G 2}
\end{array}\right]+B_{7}^{G 2} \Delta d_{G 2} \\
& +B_{2}^{G 2} \Delta v_{q d}^{G}+B_{3}^{G 2} \Delta \omega_{G 2}, \\
F_{1}^{G 2}= & B_{1}^{G 2} \frac{d_{G 2_{0}}}{2}\left[\begin{array}{ll}
1 & 0
\end{array}\right], \quad B_{7}^{G 2}=B_{1}^{G 2} \frac{V_{d c_{0}}^{G 2}}{2} .
\end{aligned}
$$

A.2. DC Part. By rearranging (5) and (10) in the frame linear differential equation

$$
\begin{gathered}
E_{2}^{G 2} \rho\left[\begin{array}{c}
\Delta V_{d c}^{G 2} \\
\Delta I_{d}^{G 2}
\end{array}\right]=A_{2}^{G_{2}}\left[\begin{array}{c}
\Delta V_{d c}^{G 2} \\
\Delta I_{d}^{G 2}
\end{array}\right]+A_{3}^{G 2} \Delta i_{q d_{i}}^{G 2} \\
+B_{4}^{G 2} \Delta d_{G 2}+B_{5}^{G 2}\left[\begin{array}{c}
\Delta E^{G 2} \\
\Delta \omega_{E}
\end{array}\right], \\
E_{2}^{G 2}=\left[\begin{array}{ll}
1 & 0 \\
0 & 1
\end{array}\right],
\end{gathered}
$$

$$
A_{2}^{G_{2}}=\left[\begin{array}{cc}
\frac{3 d_{G 2_{0}} i_{q i 0}^{G 2} X_{C_{d c G 2}} \omega_{b}}{4 V_{d c_{0}}^{G 2}} & X_{C_{d c G 2} \omega_{b}} \\
-\frac{1}{Z_{2}} & -\frac{3 X_{L_{c G 2}} \omega_{E_{0}}}{\pi Z_{2} \omega_{b}}
\end{array}\right]
$$$$
A_{3}^{G_{2}}=\left[\begin{array}{cc}
-\frac{3 d_{G 2_{0}} X_{C_{d c G 2}} \omega_{b}}{4} & 0 \\
0 & 0
\end{array}\right] \text {, }
$$

$$
\begin{gathered}
B_{4}^{G_{2}}=\left[\begin{array}{c}
3 i_{q i 0}^{G 2} X_{C_{d c G 2}} \omega_{b} \\
2 V_{d c_{0}}^{G 2} \\
0
\end{array}\right], \\
B_{5}^{G_{2}}=\left[\begin{array}{cc}
0 & 0 \\
\frac{3 \sqrt{3}}{\pi Z_{2}} & -\frac{3 I_{d_{0}}^{G 2} X_{L_{c G 2}}}{\pi Z_{2} \omega_{b}}
\end{array}\right], \\
Z_{2}=\frac{1}{\omega_{b}}\left(X_{L_{d c}}+2 X_{L_{c}}\right) .
\end{gathered}
$$

By replacing (19) and (22) into the (A.4),

$$
\begin{gathered}
E_{2}^{G 2} \rho\left[\begin{array}{c}
\Delta V_{d c}^{G 2} \\
\Delta I_{d}^{G 2}
\end{array}\right]=A_{2}^{G 2}\left[\begin{array}{c}
\Delta V_{d c}^{G 2} \\
\Delta I_{d}^{G 2}
\end{array}\right]+A_{4}^{G 2} \Delta i_{q d_{i}}^{G}+B_{4}^{G 2} \Delta d_{G 2} \\
+B_{5}^{G 2}\left[\begin{array}{c}
\Delta E^{G 2} \\
\Delta \omega_{E}
\end{array}\right]+B_{6}^{G 2} \Delta v_{q d}^{G}, \\
A_{4}^{G_{2}}=A_{3}^{G_{2}} T_{G 2}^{-1}, \quad B_{6}^{G}=A_{3}^{G_{2}} i_{\delta_{G 2}}^{0} M_{q d}^{G 2} .
\end{gathered}
$$


A.3. Differential Equation of Power Circuit

$$
\begin{aligned}
& E^{G 2} \Delta \dot{X}^{G 2}=A^{G 2} \Delta X^{G 2}+B^{G 2} \Delta U^{G 2}+C^{G 2} \Delta X_{C}^{G 2}, \\
& \Delta X^{G 2}=\left[\begin{array}{llll}
\Delta i_{q i}^{G 2} & \Delta i_{d i}^{G 2} & \Delta v_{d c}^{G 2} & \Delta I_{d}^{G 2}
\end{array}\right], \\
& E^{G 2}=\left[\begin{array}{cc}
E_{1}^{G 2} & 0 \\
0 & E_{2}^{G 2}
\end{array}\right], \quad B^{G 2}=\left[\begin{array}{cc}
B_{2}^{G 2} & 0 \\
B_{6}^{G 2} & B_{5}^{G 2}
\end{array}\right] \text {, } \\
& C^{G 2}=\left[\begin{array}{cc}
B_{3}^{G 2} & B_{7}^{G 2} \\
0 & B_{4}^{G 2}
\end{array}\right], \quad A^{G 2}=\left[\begin{array}{cc}
A_{1}^{G 2} & F_{1}^{G 2} \\
A_{4}^{G 2} & A_{2}^{G 2}
\end{array}\right] \text {. }
\end{aligned}
$$

\section{B. Control Circuit}

B.1. Active Power Droop Controller. Linear form of (11) and (13),

$$
\begin{aligned}
& \Delta \omega_{G 2}=-k_{p} \Delta \widetilde{P}, \\
& \Delta \widetilde{P}=\frac{\omega_{f}}{s+\omega_{f}} \Delta P .
\end{aligned}
$$

By combining (B.1) and (B.2),

$$
s \Delta \omega_{G 2}=-\omega_{f} \Delta \omega_{G 2}-k_{p} \omega_{f} \Delta P .
$$

B.2. Reactive Power Droop Controller. In the same way of the active power control, from (12) and (14) we have

$$
s \Delta d_{G 2}=-\omega_{f} \Delta d_{G 2}-k_{v} \omega_{f} \Delta Q .
$$

\section{B.3. Differential Equation of Control Circuit}

$$
\begin{gathered}
\Delta \dot{X}_{C}^{G 2}=A_{C}^{G 2} \Delta X_{C}^{G 2}+B_{C 0}^{G 2} \Delta U_{C}^{G 2}, \\
\Delta X_{C}^{G 2}=\left[\begin{array}{ll}
\Delta \omega_{G 2} & \Delta d_{G 2}
\end{array}\right], \\
\Delta U_{C}^{G 2}=\left[\begin{array}{ll}
\Delta P & \Delta Q
\end{array}\right], \quad A_{C}^{G 2}=\left[\begin{array}{cc}
-\omega_{f} & 0 \\
0 & -\omega_{f}
\end{array}\right] .
\end{gathered}
$$

With writing the active and reactive power in terms of voltage and current

$$
\begin{gathered}
\Delta \dot{X}_{C}^{G 2}=A_{C}^{G 2} \Delta X_{C}^{G 2}+C_{C}^{G 2} \Delta X^{G 2}+B_{C 6}^{G 2} \Delta v_{q d}^{G}, \\
C_{C}^{G 2}=\left[\begin{array}{ll}
B_{C 5}^{G 2} & 0
\end{array}\right], \quad B_{C 5}^{G 2}=B_{C 1}^{G 2} B_{C 2}^{G 2} T_{G 2}^{-1}, \\
B_{C 6}^{G 2}=B_{C 1}^{G 2} B_{C 3}^{G 2} T_{G 2}^{-1}+B_{C 4}^{G 2} M_{q d}^{G 2}, \\
B_{C 4}^{G 2}=B_{C 1}^{G 2} B_{C 2}^{G 2} i_{\delta_{G 2}}^{0}+B_{C 1}^{G 2} B_{C 3}^{G 2} v_{\delta_{G 2}}^{0}, \\
B_{C 1}^{G 2}=\left[\begin{array}{cc}
-\frac{3}{2} k_{p} \omega_{f} & 0 \\
0 & -\frac{3}{2} k_{v} \omega_{f}
\end{array}\right], \\
B_{C 2}^{G 2}=\left[\begin{array}{cc}
-v_{d 0}^{G 2} & v_{q 0}^{G 2} \\
v_{q 0}^{G 2} & v_{d 0}^{G 2}
\end{array}\right], \quad B_{C 3}^{G 2}=\left[\begin{array}{cc}
i_{d i 0}^{G 2} & -i_{q i 0}^{G 2} \\
i_{q i 0}^{G 2} & i_{d i 0}^{G 2}
\end{array}\right] .
\end{gathered}
$$

\section{Linear Differential Equations of DG (25)}

$$
\begin{aligned}
& \rho \Delta X^{\mathrm{DG}}=A^{\mathrm{DG}} \Delta X^{\mathrm{DG}}+B^{\mathrm{DG}} \Delta U^{\mathrm{DG}},
\end{aligned}
$$

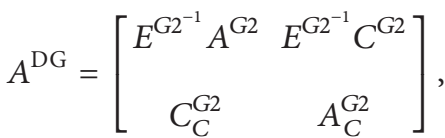

$$
\begin{aligned}
& B^{\mathrm{DG}}=\left[\begin{array}{c}
E^{G 2^{-1}} B^{G 2} \\
B_{C 7}^{G 2}
\end{array}\right], \quad B_{C 7}^{G 2}=\left[\begin{array}{ll}
B_{C 6}^{G 2} & 0
\end{array}\right] \text {. }
\end{aligned}
$$

\section{References}

[1] N. Pogaku, M. Prodanović, and T. C. Green, "Modeling, analysis and testing of autonomous operation of an inverter-based microgrid," IEEE Transactions on Power Electronics, vol. 22, no. 2, pp. 613-625, 2007.

[2] P. R. H. Lasseter and P. Paigi, "Microgrid: a conceptual solution," in Proceedings of the IEEE 35th Annual Power Electronics Specialists Conference (PESC '04), pp. 4285-4290, June 2004.

[3] E. Antônio, A. Coelho, P. C. Cortizo, P. Francisco, and D. Garcia, "Small-signal stability for parallel-connected inverters in standalone AC supply systems," IEEE Transactions on Industry Applications, vol. 38, no. 2, pp. 533-542, 2002.

[4] C. K. Sao and P. W. Lehn, "Intentional islanded operation of converter fed microgrids," in Proceedings of the IEEE Power Engineering Society General Meeting, Quebec, Canada, 2006.

[5] H. Nikkhajoei and R. Iravani, "Steady-state model and power flow analysis of electronically-coupled fistributed resource units," IEEE Transactions on Power Delivery, vol. 22, no. 1, pp. 721-728, 2007.

[6] R. Majumder, A. Ghosh, G. Ledwich, and F. Zare, "Stability analysis and control of multiple converter based autonomous microgrid," in Proceedings of the IEEE International Conference on Control and Automation (ICCA '09), pp. 1663-1668, Christchurch, New Zealand, December 2009.

[7] F. Katiraei, M. R. Iravani, and P. W. Lehn, "Small-signal dynamic model of a micro-grid including conventional and electronically interfaced distributed resources," IET Generation, Transmission and Distribution, vol. 1, no. 3, pp. 369-378, 2007.

[8] Y. Zhang, Z. Jiang, and X. Yu, "Small-signal modeling and analysis of parallel-connected voltage source inverters," in Proceedings of the IEEE 6th International Power Electronics and Motion Control Conference (IPEMC '09), pp. 377-383, Wuhan, China, 2009.

[9] P. C. Krause, O. Wasynczuk, and S. D. Sudhoff, Analysis of Electric Machinery and Drive Systems, John Wiley \& Sons, New York, NY, USA, 2002.

[10] S. C. Vegunta, J. V. Milanović, and S. Z. Djokić, "Modelling of $\mathrm{VHz}$ and vector controlled ASDs in PSCAD/EMTDC for voltage sag studies," Electric Power Systems Research, vol. 80, no. 1, pp. 1-8, 2010.

[11] P. Kundur, Power System Stability and Control, McGraw-Hill, New York, NY, USA, 1994. 


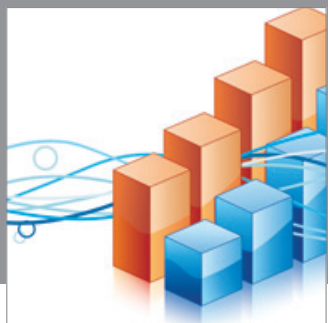

Advances in

Operations Research

mansans

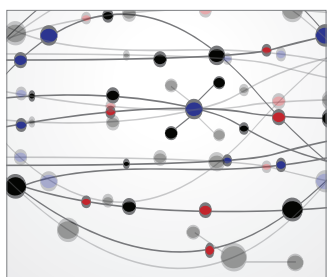

The Scientific World Journal
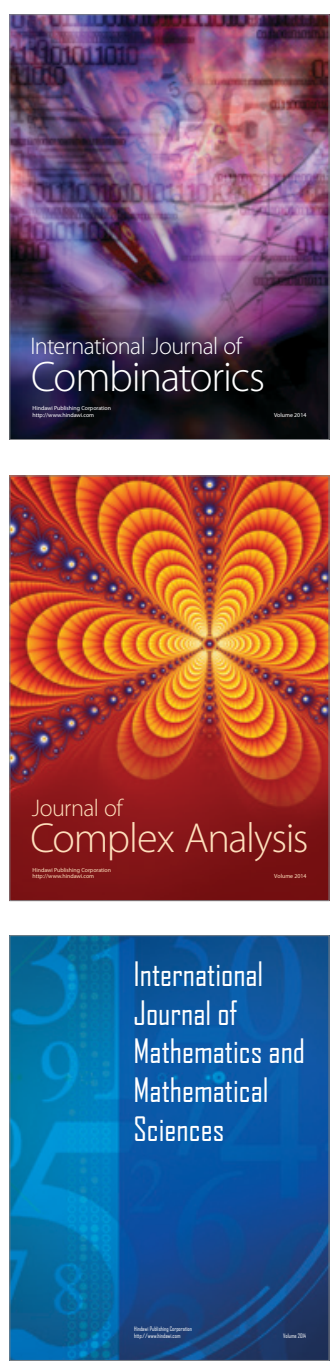
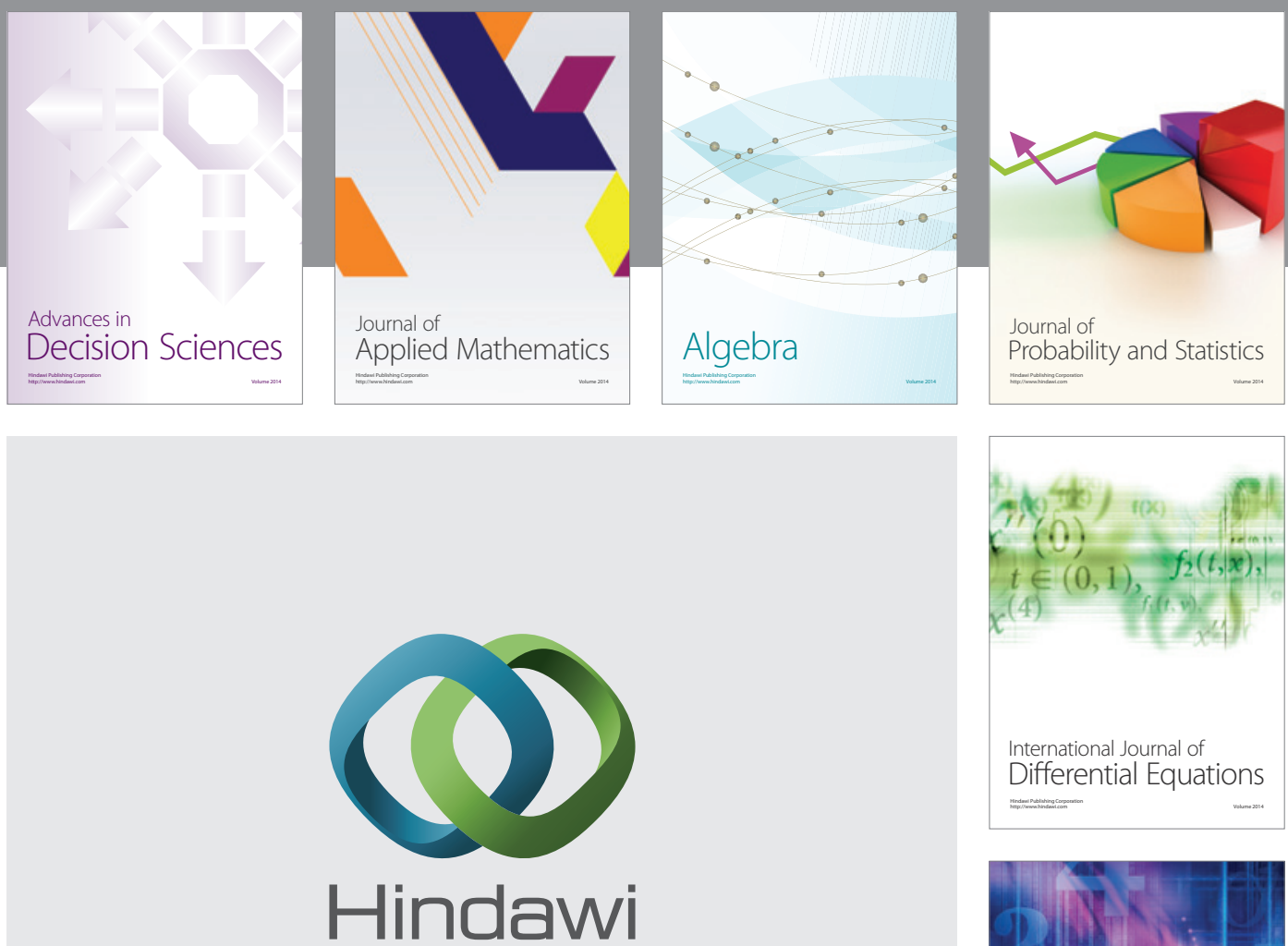

Submit your manuscripts at http://www.hindawi.com
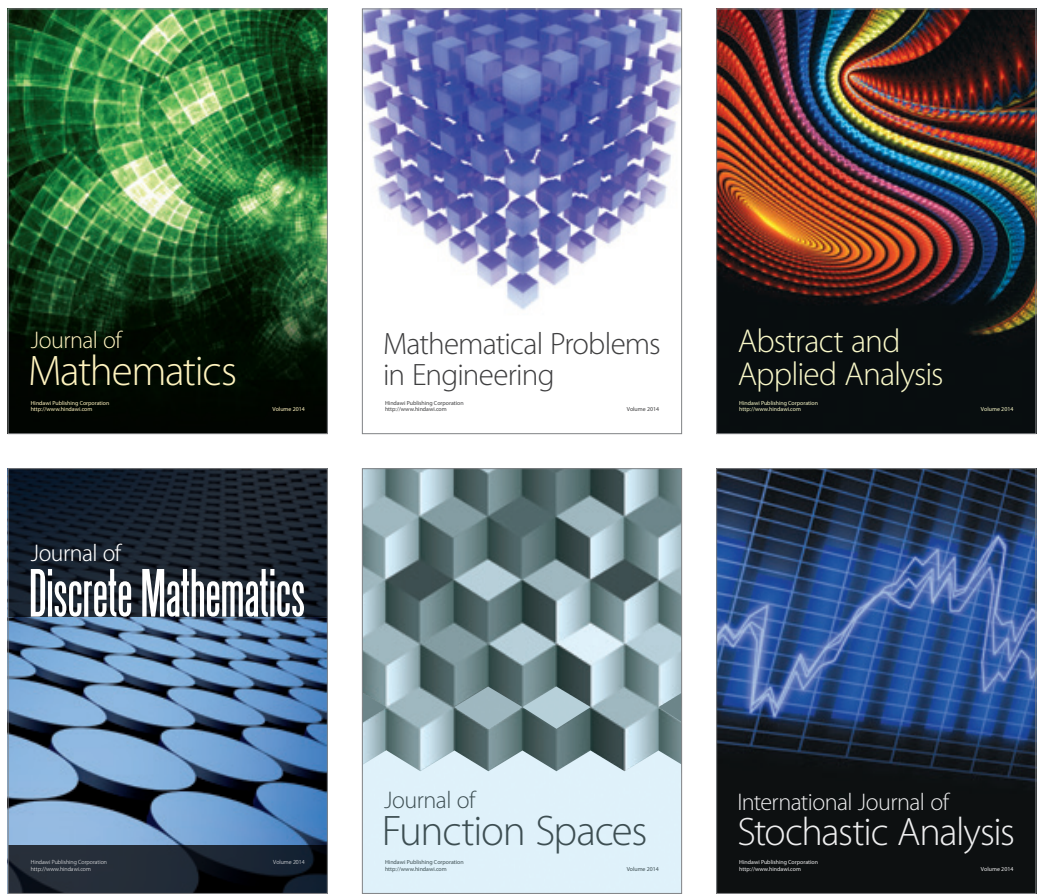

Journal of

Function Spaces

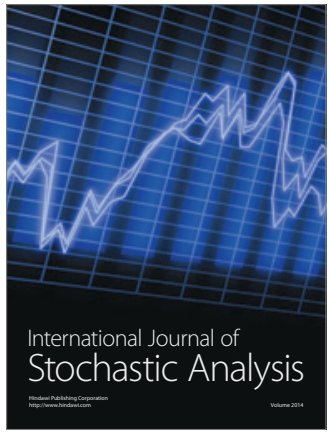

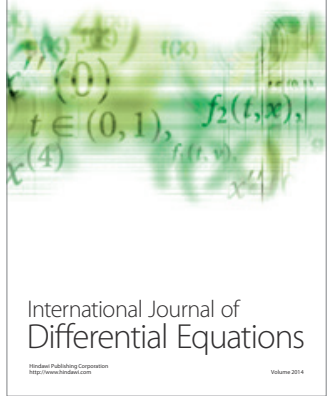
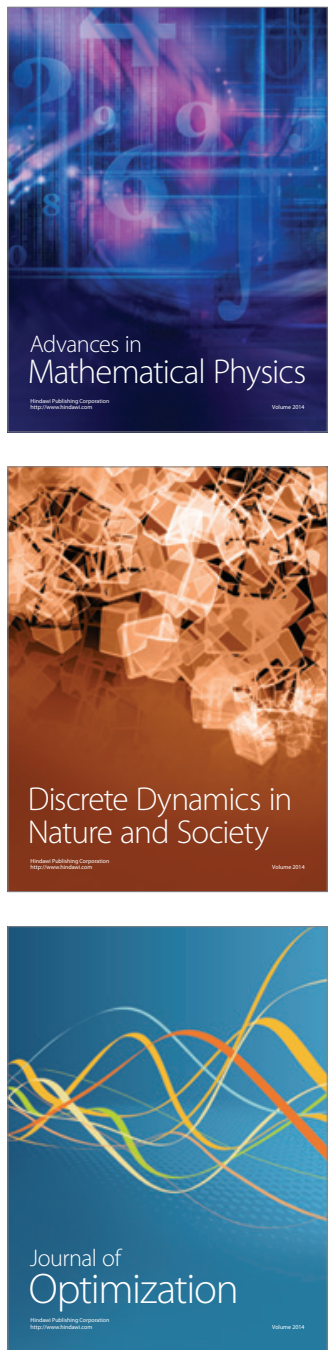